\title{
Serum microRNA 125b as a diagnostic or prognostic biomarker for advanced NSCLC patients receiving cisplatin-based chemotherapy
}

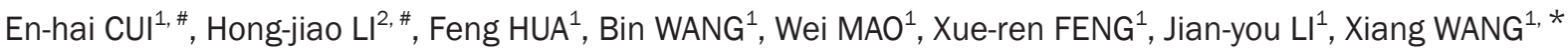 \\ ${ }^{1}$ Respiratory Medicine, Huzhou Central Hospital, Huzhou 313000, China; ${ }^{2}$ Department of Stomatology, Changhai Hospital, Second \\ Military Medical University, Shanghai 200433, China
}

\begin{abstract}
Aim: To investigate the expression profile of microRNAs in inoperable advanced non-small cell lung cancer (NSCLC) patients receiving chemotherapy and the potential relevance of microRNAs to clinicopathological characteristics and prognosis.

Methods: Serum samples were taken from 260 inoperable advanced NSCLC patients and 260 healthy individuals. All the patients received cisplatin-based chemotherapy, including NP/NC regimens, GP/GC regimens, and TP/TC regimens. The serum levels of microRNAs (miR-125b, miR-10b, miR-34a and miR-155) were determined by quantitative real-time PCR.

Results: Serum levels of the 4 microRNAs examined in NSCLC patients were significantly increased as compared with healthy individuals. The levels of miR-125b and miR-155 were changed in a similar pattern: the patients with stage IV disease had the highest one, while the patients with stage III A and stage III B disease showed similar increased levels. The levels of miR-10b and miR-34a in the patients with different stages were increased to similar extent. The level of miR-125b in poorly differentiated cancer was significantly higher than those in well and moderately differentiated cancers, while the levels of miR-10b, miR-34a, and miR-155 did not significantly differ with cancer differentiation. Among the 4 microRNAs examined, only miR-125b was significantly associated with therapeutic response, exhibiting higher expression levels in non-responsive patients. Furthermore, the high level of miR-125b was significantly correlated with poor patient survival. A multivariate Cox regression analysis showed that the expression level of miR-125b was an independent prognostic marker in NSCLC patients.

Conclusion: Our results suggest that miR-125b is a potential diagnostic or prognostic biomarker for NSCLC. This finding has important implications for development of targeted therapeutics to overcome chemotherapeutic resistance in NSCLC.
\end{abstract}

Keywords: microRNA 125b; biomarker; non-small cell lung cancer (NSCLC); cisplatin-based chemotherapy; prognosis

Acta Pharmacologica Sinica (2013) 34: 309-313; doi: 10.1038/aps.2012.125; published online 17 Sep 2012

\section{Introduction}

Lung cancer is currently the most widespread cancer worldwide ${ }^{[1]}$. Non-small cell lung cancer (NSCLC) accounts for $80 \%$ of all lung cancers. Although there has been some progress in the treatment of NSCLC in recent years, patient prognosis remains very poor, particularly for advanced NSCLC ${ }^{[2-4]}$. Chemotherapy is an important component in the treatment paradigm for NSCLC. Cisplatin-based chemotherapy is the first line of treatment for advanced inoperable NSCLC; however, the chemotherapy response rate varies among patients ${ }^{[5-8]}$. The mechanisms accounting for the different responses to chemotherapy in NSCLC patients remain

\footnotetext{
\# These authors contributed equally to this work.

* To whom correspondence should be addressed.

E-mail wangxiang20120531@gmail.com

Received 2012-06-01 Accepted 2012-08-02
}

unclear. There is no validated sensitivity/resistance predictive marker available in the clinical setting. The identification of predictive markers for chemotherapy response is clinically warranted to further improve the efficacy of chemotherapy treatment in advanced NSCLC.

MicroRNAs (miRNAs) represent a class of small non-coding RNAs. These single-stranded molecules have a length of 19-23 nucleotides and regulate gene expression at the posttranscriptional level, thus controlling crucial physiological processes through three mechanisms: binding to complementary sequences on target messenger RNA transcripts (mRNAs), repressing mRNA translation and cleaving target mRNA $^{[9,10]}$. Furthermore, increasing evidence shows that miRNAs can act as oncogenes or tumor suppressors and may play important roles in tumorigenesis ${ }^{[11-13]}$. Therefore, miRNA expression profiles may become useful biomarkers for cancer diagnosis, prognosis and prediction of treatment-response 
and may serve as powerful tools for cancer prevention and therapy ${ }^{[14-19]}$. In recent years, some miRNAs have also been reported to be involved in drug-resistance in diseases such as breast cancer ${ }^{[3]}$, chronic lymphocytic leukemia ${ }^{[20]}$, metastatic colorectal cancer ${ }^{[21]}$ and esophageal cancer ${ }^{[22]}$. A recent study investigated the association between the expression of several miRNAs and chemotherapeutic resistance in breast cancer. The author found that only miR-125b was significantly associated with a therapeutic response, exhibiting higher expression levels in non-responsive patients. This finding suggests that miR-125b is a good biomarker for chemotherapy response in cancer patients ${ }^{[3]}$. To date, there has been no report on the role of circulating miRNAs in the chemotherapy response status and prognosis of NSCLC.

The aim of this study was to clarify these issues in a Chinese cohort with advanced NSCLC receiving cisplatin-based chemotherapy. In this study, we measured the serum levels of circulating miRNAs in advanced inoperable NSCLC patients receiving chemotherapy and investigated the relationship between circulating miRNA expression and clinical features, chemotherapeutic response and prognosis.

\section{Materials and methods Patients}

Patients received cisplatin-based chemotherapy treatment for NSCLC between January 2009 and December 2011 from Huzhou Central Hospital. A total of 260 patients, including 99 stage III A, 101 stage III B and 60 stage IV patients, were enrolled in this study. Before starting the treatment, the patients underwent a baseline blood draw for miRNA expression profiling. To avoid the potential influence of poor clinical conditions on chemotherapy response, other eligibility criteria included the following: normal blood chemistry (hemoglobin $>0.9 \mathrm{~g} / \mathrm{L}$, neutrophil count $>1500 / \mathrm{mm}^{3}$ and platelet count $>100000 / \mathrm{mm}^{3}$ ), normal hepatic (bilirubin $<1.5$ times the normal upper limit, aspartate aminotransferase and alanine aminotransferase $<2$ times the normal upper limit) and renal (Creatine clearance rate $>50 \mathrm{~mL} / \mathrm{s}$ ) functions and a normal electrocardiogram at the beginning of treatment. The exclusion criteria included symptomatic brain metastases, spinal cord compression, uncontrolled massive pleural effusion, previous chemotherapy treatment and other chronic disease. In addition, 260 age- and sex-matched healthy adults with no history of cancer were recruited as controls. Serum samples were processed within $2-5 \mathrm{~h}$ after collection. First, the serum was separated by centrifugation at 4000 rounds per minute for $10 \mathrm{~min}$, and then, potential contaminating cells were removed by a $13-\mathrm{mm}$ serum filter (Thermo Fisher Scientific Inc, USA). The samples were divided into aliquots and immediately cryopreserved at $-80^{\circ} \mathrm{C}$ until use. The study was approved by the ethics committees of Huzhou Central Hospital, and written informed consent was obtained from each participant.

\section{Chemotherapy regimens and therapeutic effect evaluation}

All patients received platinum-based chemotherapy: 18 (6.9\%) received NP/NC regimens (vinorelbine plus cisplatin/carbo- platin), 132 (50.8\%) received GP/GC regimens (gemcitabine plus cisplatin/carboplatin), and $110(42.3 \%)$ received TP/TC regimens (taxol/docetaxel plus cisplatin/carboplatin). The chemotherapy protocol and dosage regimens were previously described $^{[23]}$. Patients achieving a complete response (CR) or partial response (PR) were considered responders. Nonresponders were categorized as either disease stabilization (SD) or disease progression (PD).

\section{Extraction of total RNA and real-time PCR}

MiRNA profiling was performed using serum samples derived from the 260 patients and 260 healthy individuals. Total RNA was isolated with TRIzol LS reagent (Invitrogen, San Diego, CA, USA) according to the manufacturer's instructions. The RNA extraction was performed using acid-phenol and chloroform, and the precipitation was carried out using ethanol and a filter cartridge. The extracted RNA was eluted in $100 \mathrm{~mL}$ of preheated Elution Solution and measured on a NanoDrop ND-1000 spectrophotometer (Thermo Fisher Scientific Inc, USA). The RNA samples were immediately stored at $-80^{\circ} \mathrm{C}$ until use.

For quantitative real-time PCR, miRNA-specific TaqMan MicroRNA Assays (Applied Biosystems, Foster City, CA, USA) for miR-16 (reference miR), miR-10b, miR-34a, miR$125 \mathrm{~b}$, and miR-155 were performed as described by the manufacturer. Briefly, $100 \mathrm{ng}$ of total RNA was reverse transcribed using primers specific to each miRNA target, followed by realtime PCR on a 7900 HT Fast Real-Time PCR System using TaqMan miRNA primers and probes (Applied Biosystems, Foster City, CA, USA). Triplicate samples, validated endogenous controls and interassay controls were used throughout. MiRNA expression levels were calculated by the $\Delta \mathrm{C}_{\mathrm{t}}$ method: $\Delta \mathrm{C}_{\mathrm{t}}=$ mean value $\mathrm{C}_{\mathrm{t}}$ (reference miR-16)-mean value $\mathrm{C}_{\mathrm{t}}$ (miRNA of interest). The relative expression of the miRNA of interest corresponded to the $2^{\Delta \mathrm{Ct}}$ value ${ }^{[4]}$.

\section{Statistical analyses}

Statistical analyses were performed with SPSS (Statistical Package for the Social Sciences) software, version 16.0. The data were presented as the mean \pm standard deviation (SD), and either the chi-square analysis or the two-tailed Student's $t$-test was used to assess the association between microRNAs in the serum and clinical features, chemotherapeutic response and NSCLC prognosis. The correlation between overall survival and serum miRNA levels was analyzed using the KaplanMeier method and the log-rank test. A multivariate analysis of the independent prognostic factors was conducted using the Cox proportional hazards model. All statistical tests were two-sided, and a $P$ value of $<0.05$ was considered statistically significant.

\section{Results}

\section{Patient characteristics}

Among the 260 patients with NSCLC, 198 were male and 62 were female. The mean age and sex distributions of the NSCLC subjects were similar between the NSCLC patients 
Table 1. Patient characteristics.

\begin{tabular}{|c|c|c|c|c|c|}
\hline & NSCLC & & Control & & $P$ value \\
\hline Age (years) & $56.45 \pm 3.4$ & & $56.31 \pm 2.1$ & & NS \\
\hline Gender (male, \%) & 198 & $76.15 \%$ & 198 & $76.15 \%$ & NS \\
\hline \multicolumn{6}{|l|}{ Smoking status } \\
\hline Smoker & 176 & $67.69 \%$ & 90 & $34.62 \%$ & $<0.001$ \\
\hline Nonsmoker & 84 & $32.31 \%$ & 170 & $65.38 \%$ & \\
\hline miR-34a & $3.08 \pm 0.23$ & & $1.08 \pm 0.31$ & & $<0.001$ \\
\hline miR-10b & $2.72 \pm 0.23$ & & $0.89 \pm 0.19$ & & $<0.001$ \\
\hline miR-155 & $4.33 \pm 0.26$ & & $1.33 \pm 0.46$ & & $<0.001$ \\
\hline \multicolumn{6}{|l|}{ Stage } \\
\hline III A & 99 & $38.08 \%$ & & & \\
\hline Squamous cell carcinoma & 101 & $38.85 \%$ & & & \\
\hline Large cell carcinoma & 32 & $12.31 \%$ & & & \\
\hline Other types & 10 & $3.85 \%$ & & & \\
\hline \multicolumn{6}{|l|}{ Differentiation } \\
\hline Well & 89 & $34.23 \%$ & & & \\
\hline Moderate & 97 & $37.31 \%$ & & & \\
\hline Poor & 74 & $28.46 \%$ & & & \\
\hline
\end{tabular}

and the controls (Table 1). NSCLC patients had a significantly higher smoking rate than controls $(P<0.001)$. The expression of miR-125b, miR-34a, miR-10b, and miR-155 was compared between NSCLC patients and the control group, and statistical significance was calculated as described in the Materials and methods section. Details are shown in Table 1.

Table 1 depicts the correlation between the circulating miRNA levels in the 260 patients, who had advanced NSCLC and received cisplatin-based chemotherapy, and clinical factors. The level of miR-125b was significantly higher in patients with late stage disease (stage IV, $P<0.001$ ) but similar in patients with stage III A and stage III B disease $(P=0.065)$ (Figure 1). A similar trend was observed in miR-155 expression among patients with stage III A, III B, and IV disease. The level of miR-155 in stage IV patients was significantly higher than in patients with stage III A and III B disease $(P<0.01$, Figure 1). Circulating miR-10b and miR-34a levels did not differ significantly in each comparison.

We next investigated whether miRNA levels were affected by tumor status (Figure 2). The level of miR-125b in poorly differentiated cancer was higher than that in well and moderately differentiated cancers $(P=0.0021, P=0.0015$, respectively). The levels of miR-10b, miR-34a, and miR-155 did not significantly differ according cancer differentiation. All of the levels of the various miRNAs were similar by histological type of cancer, including adenocarcinoma, squamous cell carcinoma, large cell carcinoma and others.

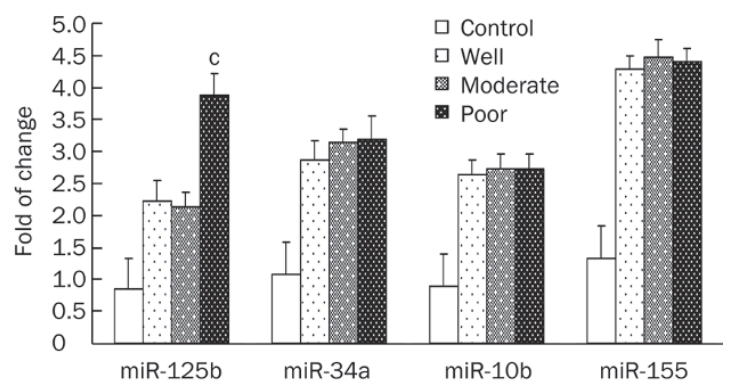

Figure 2. The miR expression levels according the tumor differentiation status. ${ }^{\mathrm{C}} P<0.01$ vs III A.
Figure 1. The miR relative expression levels according the cancer stages. ${ }^{\mathrm{c}} P<0.01$ vs III A. 
Circulating miRNA expression levels and chemotherapeutic response

To determine whether the circulating miRNA expression levels were associated with chemotherapeutic efficacy, therapeutic response was evaluated according to the Radiologic Response Evaluation Criteria in Solid Tumors (RECIST) after chemotherapy. According to RECIST, 99 patients (38\%) responded to chemotherapy with PR or CR; 161 (64\%) patients were not responsive with SD or PD. Among the circulating miRNAs, only miR-125b was significantly associated with therapeutic response, exhibiting significantly higher expression levels in non-responsive patients $(P=0.003$, Table 2$)$. The other miRNA expression levels were similar between responders and nonresponders $(P>0.05)$.

Table 2. Circulating miR expression levels and chemotherapeutic response.

\begin{tabular}{lrrrrr}
\hline $\begin{array}{l}\text { Treatment } \\
\text { response }\end{array}$ & No & miR-125b & miR-34a & miR-10b & miR-155 \\
\hline CR+PR & 99 & $2.27 \pm 0.33$ & $3.04 \pm 0.21$ & $2.70 \pm 0.19$ & $4.21 \pm 0.23$ \\
SD+PD & 161 & $3.07 \pm 0.38$ & $3.11 \pm 0.26$ & $2.73 \pm 0.24$ & $2.42 \pm 0.27$ \\
\hline
\end{tabular}

CR, complete response; PR, partial response; SD, stable disease; PD, disease progression.

\section{Circulating miRNA expression levels and overall survival}

According to the mean miRNA expression levels shown in Table 1, all NSCLC subjects could be divided into either high expression (including those whose miRNA levels were higher than their respective mean miRNA expression levels) or low expression groups (including those whose miRNA levels were lower than their respective mean miRNA expression levels). A Kaplan-Meier survival analysis revealed that high expression of serum miR-125b was significantly correlated with poor patient survival $(P=0.0012$, Figure 3$)$. Patients with

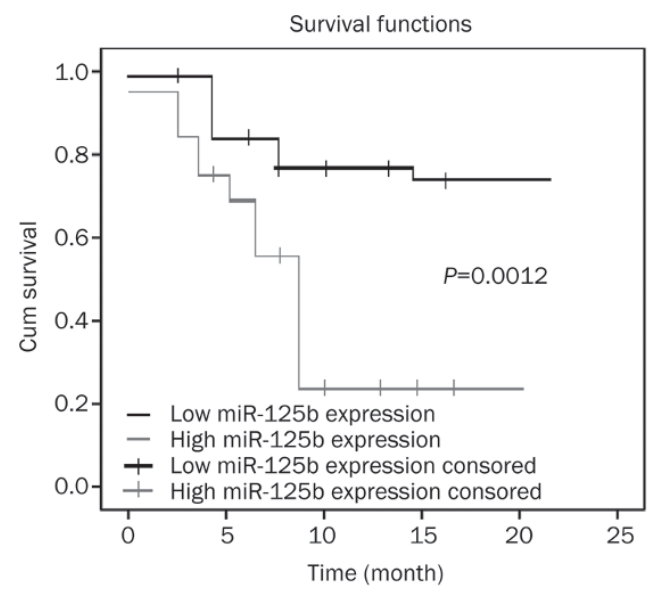

Figure 3. TKaplan-Meier survival analysis according to miR-125b expression levels. high expression of serum miR-155 had poorer survival rates than those with low expression $(P=0.052)$. There were no significant differences between the high expression groups and the low expression groups for circulating miR-10b and miR-34a. A multivariate Cox regression analysis showed that the expression level of miR-125b was an independent prognostic marker in NSCLC patients $(\mathrm{HR}=2.03$; 95\% CI, 1.14-3.05; $P=0.0013$ ), while miR-155 was not. The other factors responsible for NSCLC prognosis included chemotherapy response $(\mathrm{HR}=4.12 ; 95 \% \mathrm{CI}, 3.64-5.84 ; P<0.001)$, cancer stage $(\mathrm{HR}=2.12$; 95\% CI, 1.89-3.76; $P<0.001)$ and differentiation status $(\mathrm{HR}=1.89$; 95\% CI, 1.34-2.54; $P=0.015$ ) (Table 3).

Table 3. The hazards ration of overall survival by multivariate Cox regression analysis.

\begin{tabular}{lccc}
\hline \multicolumn{1}{c}{ Factors } & HR & $95 \% \mathrm{Cl}$ & $P$ value \\
\hline miR-125b expression & 2.03 & $1.14-3.05$ & 0.0013 \\
Chemotherapy response & 4.12 & $3.64-5.84$ & $<0.001$ \\
Cancer stage & 2.12 & $1.89-3.76$ & $<0.001$ \\
Differentiation status & 1.89 & $1.34-2.54$ & 0.015 \\
\hline
\end{tabular}

CR, complete response; PR, partial response; SD, stable disease; PD, disease progression; HR, hazard ratio.

\section{Discussion}

MiRNAs regulate gene expression by binding to and modulating the translation of specific miRNAs. We investigated the possible association between several circulating miRNAs, including miR-125b, miR-10b, miR-34a, and miR-155, and clinical features, chemotherapeutic response and prognosis in advanced inoperable NSCLC patients receiving chemotherapy. While miR-125b expression in tumor tissues has been studied in various cancers, the expression of circulating miR$125 \mathrm{~b}$ has not been reported in NSCLC.

In this study, circulating miR-125b was significantly associated with cancer stage, tumor differentiation status and therapeutic response. Patients with high expression of serum miR-155 had poorer survival. Our results suggest a possible role for miR-125b as a circulating predictor of chemotherapy response and prognosis after chemotherapy in NSCLC patients. To the best of our knowledge, this is the first report regarding the role of miR-125b in NSCLC patients.

MiR-125b has been reported to have complicated oncogenelike or tumor suppressor-like functions in different cancer types or cell lines. A previous study showed that circulating miR-125b expression correlated with chemotherapeutic resistance in breast cancer patients ${ }^{[3]}$. In that study, higher circulating miR-125b levels were correlated with adjuvant chemotherapy resistance; the higher the level of $\mathrm{miR}-125 \mathrm{~b}$ expression, the greater the resistance ${ }^{[3]}$. Another study showed that miR-125b conferred cisplatin resistance to ovarian cancer cells by targeting the pro-apoptotic Bcl-2 antagonist killer ${ }^{[5]}$. In addition, upregulation of miR-125b expression contributed to cisplatin 
resistance. This finding suggests that miR-125b is not only a marker for chemotherapeutic response but can also be used as a target for targeted therapeutics designed to overcome cisplatin resistance in ovarian cancer ${ }^{[5]}$.

Our data show that circulating miR-125b levels affect the chemotherapy response and prognosis in NSCLC patients. The mechanism of how miR-125b affects chemotherapy resistance/response has not been fully elucidated. Current evidence suggests that miR-125b affects cell apoptosis and proliferation, because upregulation of miR-125b causes marked inhibition of cisplatin-induced apoptosis and subsequently increases the resistance to cisplatin ${ }^{[19,24,25]}$.

Ectopic miR-34a expression has also been shown to result in cell cycle arrest, growth inhibition and attenuated chemoresistance to the anticancer drug camptothecin by inducing apoptosis, suggesting that miR-34a has a potential role in the treatment of p53-defective prostate cancer ${ }^{[26]}$. However, current clinical studies of NSCLC and our present study do not support this hypothesis. A possible explanation is that the effect of miR-34a as an anti-tumor agent is cancer type-specific.

Our study does have some limitations. First, we only enrolled patients with advanced NSCLC, and early stage I and stage II NSCLC patients were excluded. Therefore, the role of miR-125b in NSCLC was only partially investigated. Second, we did not carry out in vitro studies to further explore the molecular mechanism under which miR-125b influences the chemotherapy response in NSCLC. Therefore, further in vivo and in vitro functional and clinical studies would be carried out in our later work.

\section{Acknowledgements}

This work was supported by Zhejiang Province Welfare Technology Applied Research Program (2011C33051) and the National Natural Science Foundation of China (№ 31171024).

\section{Author contribution}

En-hai CUI conducted the experiment data and wrote the article; Hong-jiao LI analyzed the data; Xiang WANG designed the experiment; and the other person helped to collect the sample and clinical data.

\section{References}

1 Neville A. Lung cancer. Clin Evid 2005; (14): 1903-20.

2 Bernal Bernal R, Leon Jimenez A, Jaen Olasolo J, Benitez Rodriguez E, Mateo Vallejo F. Local recurrence after non-small cell lung cancer surgery: prognosis variables. An Med Interna 2008; 25: 55-60.

3 Ichinose Y, Tsuchiya R, Yasumitsu T, Koike T, Yamato Y, Nakagawa K, et al. Prognosis of non-small cell lung cancer patients with positive pleural lavage cytology after a thoracotomy: results of the survey conducted by the Japan Clinical Oncology Group. Lung Cancer 2001; 31: $37-41$.

4 Okada A, Hirono T, Watanabe T. Safety and prognosis of limited surgery for octogenarians with non-small-cell lung cancer. Gen Thorac Cardiovasc Surg 2012; 60: 97-103.

5 Pathak AK, Bhutani M, Mohan A, Guleria R, Bal S, Kochupillai V. Non small cell lung cancer (NSCLC): current status and future prospects. Indian J Chest Dis Allied Sci 2004; 46: 191-203.
6 Okamoto H, Watanabe K. Medical treatment for stage III non-smallcell lung cancer (NSCLC). Gan To Kagaku Ryoho 2007; 34: 841-8.

7 Brockmoller J, Junker K, Multhoff G. New perspectives in the management of non-small-cell lung carcinoma (NSCLC). Onkologie 2006; 29: 25-8.

8 Chang A. Chemotherapy, chemoresistance and the changing treatment landscape for NSCLC. Lung Cancer 2011; 71: 3-10.

9 Huppi K, Volfovsky N, Mackiewicz M, Runfola T, Jones TL, Martin SE, et al. MicroRNAs and genomic instability. Semin Cancer Biol 2007; 17: $65-73$.

10 Leung AK, Sharp PA. Function and localization of microRNAs in mammalian cells. Cold Spring Harb Symp Quant Biol 2006; 71: 29-38.

11 Bagnyukova TV, Pogribny IP, Chekhun VF. MicroRNAs in normal and cancer cells: a new class of gene expression regulators. Exp Oncol 2006; 28: 263-9.

12 Osada H, Takahashi T. MicroRNAs in biological processes and carcinogenesis. Carcinogenesis 2007; 28: 2-12.

13 Pfeffer S, Voinnet O. Viruses, microRNAs and cancer. Oncogene 2006; 25: 6211-9.

14 Cortez MA, Welsh JW, Calin GA. Circulating microRNAs as noninvasive biomarkers in breast cancer. Recent Results Cancer Res 2012; 195: 151-61.

15 Lovat F, Valeri N, Croce CM. MicroRNAs in the pathogenesis of cancer. Semin Oncol 2011; 38: 724-33.

16 Ng EK, Chong WW, Jin H, Lam EK, Shin VY, Yu J, et al. Differential expression of microRNAs in plasma of patients with colorectal cancer: a potential marker for colorectal cancer screening. Gut 2009; 58: 1375-81.

17 Wei J, Xie L, Taron M, Rosell R, Liu B. Epigenetic alterations of tumor marker microRNAs: towards new cancer therapies. Drug News Perspect 2010; 23: 655-61.

18 Yu DC, Li QG, Ding XW, Ding YT. Circulating microRNAs: potential biomarkers for cancer. Int J Mol Sci 2011; 12: 2055-63.

19 Wang H, Tan G, Dong L, Cheng L, Li K, Wang Z, et al. Circulating miR$125 \mathrm{~b}$ as a marker predicting chemoresistance in breast cancer. PLoS One 2012; 7: e34210.

20 Ferracin M, Zagatti B, Rizzotto L, Cavazzini F, Veronese A, Ciccone $\mathrm{M}$, et al. MicroRNAs involvement in fludarabine refractory chronic lymphocytic leukemia. Mol Cancer 2010; 9: 123.

21 Graziano F, Canestrari E, Loupakis F, Ruzzo A, Galluccio N, Santini $D$, et al. Genetic modulation of the Let-7 microRNA binding to KRAS 3'-untranslated region and survival of metastatic colorectal cancer patients treated with salvage cetuximab-irinotecan. Pharmacogenomics J 2010; 10: 458-64.

22 Mathe EA, Nguyen GH, Bowman ED, Zhao Y, Budhu A, Schetter AJ, et al. MicroRNA expression in squamous cell carcinoma and adenocarcinoma of the esophagus: associations with survival. Clin Cancer Res 2009; 15: 6192-200.

23 Feng J, Sun X, Sun N, Qin S, Li F, Cheng H, et al. XPA A23G polymorphism is associated with the elevated response to platinum-based chemotherapy in advanced non-small cell lung cancer. Acta Biochim Biophys Sin 2009; 41: 429-35.

24 Yang YQ, Li L, Wang J, Liu XY, Chen XZ, Zhang W, et al. A novel GATA4 loss-of-function mutation associated with congenital ventricular septal defect. Pediatr Cardiol 2012; 33: 539-46.

25 Kong F, Sun C, Wang Z, Han L, Weng D, Lu Y, et al. miR-125b confers resistance of ovarian cancer cells to cisplatin by targeting proapoptotic Bcl-2 antagonist killer 1. J Huazhong Univ Sci Technolog Med Sci 2011; 31: 543-9.

26 Fujita Y, Kojima K, Hamada N, Ohhashi R, Akao Y, Nozawa Y, et al. Effects of miR-34a on cell growth and chemoresistance in prostate cancer PC3 cells. Biochem Biophys Res Commun 2008; 377: 114-9. 